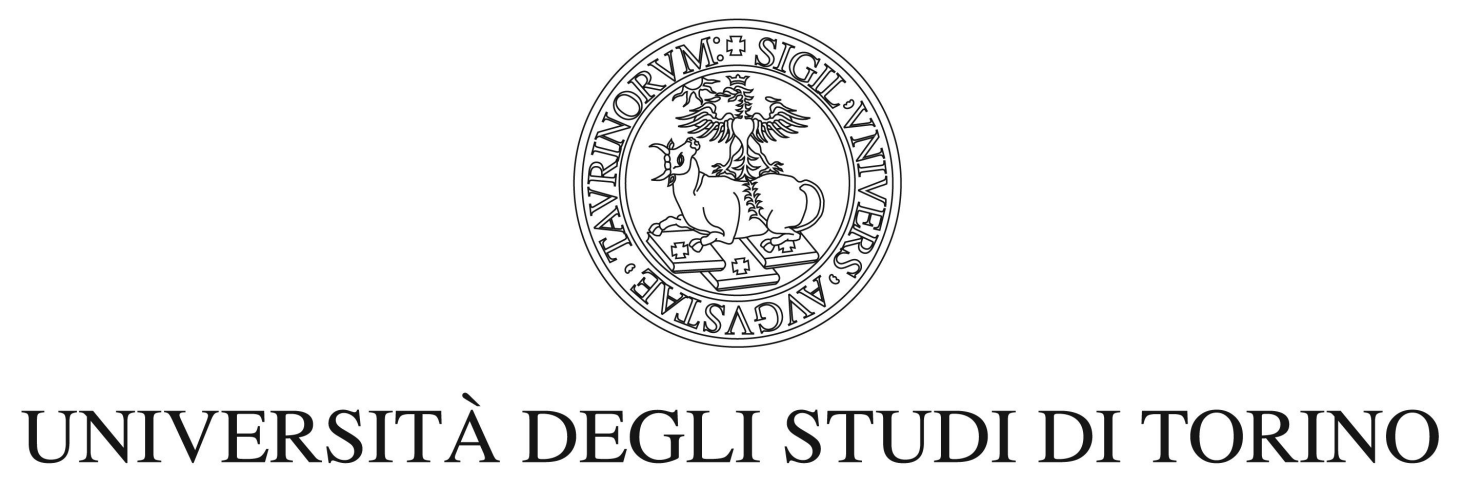

This accepted author manuscript is copyrighted. Changes resulting from the publishing process - such as editing, corrections, structural formatting, and other quality control mechanisms - are not reflected in this version of the text. For any quotation, please refer to the definitive version published in:

«Journal of Jewish Thought and Philosophy», XXI (2013), n. 1, pp. 57-71 DOI: http://dx.doi.org/10.1163/1477285X-12341242 


\title{
DIE FRAGE „WAS IST?“ BEI HERMANN COHEN UND FRANZ ROSENZWEIG“
}

\author{
Luca Bertolino \\ University of Turin \\ Department of Philosophy and Education \\ luca.bertolino@unito.it
}

\begin{abstract}

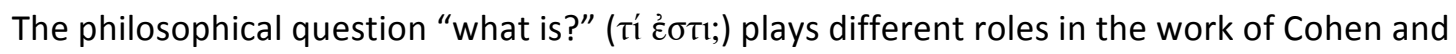
Rosenzweig. According to Cohen, it expresses the authentic meaning of the Socratic concept, which has its methodical-transcendental foundation in the Platonic Idea as answer, since it gives an account of the concept. So Cohen turns the question into an epistemological problem, because it ultimately refers to the necessary condition of knowledge. In contrast, Rosenzweig sees in the "what is?" question grounds to condemn the "old" philosophy founded on the identity of being and thought. In his view, the question is the original sin of the "philosophy of the All", which has always reduced everything to something completely different by means of the altering word "is" in the "is"-question. Nevertheless, with regard to the "what is?" question, it is possible to detect a kind of agreement between the two philosophers: namely, Rosenzweig opposes a claim of ontological reduction that Cohen also rejects.
\end{abstract}

\footnotetext{
Revidierte Fassung eines anlässlich der internationalen Konferenz „Vom „System` zum „Stern“. Hermann Cohen und Franz Rosenzweig auf dem Weg zu einer neuen Kultur der Vernunft“" (Evangelische Akademie Arnoldshain, Schmitten, 25.-27. November 2005) gehaltenen Vortrags. Die Konferenz wurde von der Hermann-Cohen-Akademie für Religion, Wissenschaft und Kunst veranstaltet, deren Direktorin, Rabbinerin Prof. Dr. Eveline Goodman-Thau, ich für die freundliche Erlaubnis zur Vorabpublikation des Textes danke. Außerdem möchte ich mich an dieser Stelle bei PD Dr. Hartwig Wiedebach für seine sorgfältige Korrektur des Manuskripts bedanken.
} 
Keywords

Hermann Cohen

Franz Rosenzweig

"What is?" question

Idealism

Ontology 
Dass die Frageform („Was?“, „Warum?“, „Wie?“, usw.) das philosophische Denken charakterisiert, ist eine einfache Beobachtung. Das Fragen drückt die Suche nach Wissen, d.h. ein Interesse an dem, was man noch nicht weiß, aus. Selbst wenn man von den Dialogen Platons oder von den Quaestiones disputatae des Thomas von Aquin absieht, findet man selten philosophische Texte, in denen der Autor nicht von einer Frage ausgeht oder auf eine Frage zurückgreift, um seinen Gedankengang zu entwickeln. Mit diesem Beitrag möchte ich die Aufmerksamkeit auf eine Frage lenken, die im Laufe der

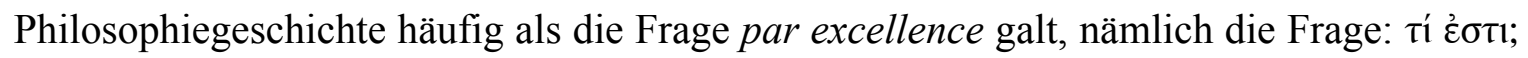
- „Was ist?“. Im Folgenden sollen die Deutungen verglichen werden, die ihr Hermann Cohen und Franz Rosenzweig gegeben haben. Die beiden Philosophen scheinen mir einander in ihrer Beziehung als Meister und Schüler sehr nahe zu stehen, in ihren theoretischen Konzeptionen dagegen relativ weit voneinander entfernt.

Cohen handelt von der Frage „Was ist?“ in der Logik der reinen Erkenntnis, und zwar dort, wo es um die Möglichkeitsbedingungen des Faktums der mathematischen Naturwissenschaft geht. Er identifiziert diese ursprünglich sokratische Frage mit dem Konzept des „Begriffs“ als solchem:

Das ist der tiefe, ewige Sinn, in welchem Sokrates seinen Begriff als die Frage: Was ist?

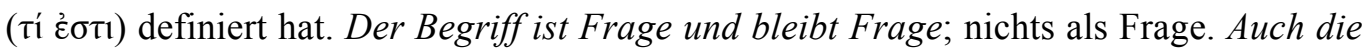
Antwort, die er enthält, muß eine neue Frage sein, eine neue Frage wecken. ${ }^{1}$

\footnotetext{
${ }^{1}$ Hermann Cohen, System der Philosophie. Erster Teil. Logik der reinen Erkenntnis (1908, 2. Aufl. 1914), Einleitung von Helmut Holzhey, Bd. 6 der Werke, hrsg. vom Hermann-Cohen-Archiv am Philosophischen Seminar der Universität Zürich unter der Leitung von Helmut Holzhey (Hildesheim/New York: Georg Olms, 1977), 378.
} 
Wenn einerseits der „Begriff“ von Sokrates als „,das große Fragezeichen des Seins: Was

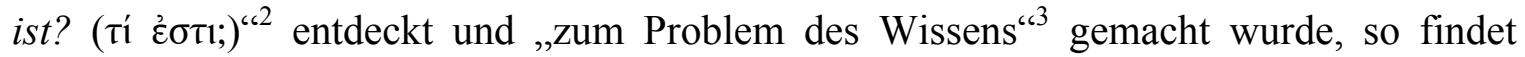
Cohen andererseits in der so genannten „Idee“ die im rechtlichen Sinne verstandene „Rechenschaft“ dieser Frage und damit zugleich „die tiefere Antwort“4 auf sie. Diese „Idee“ ist aber keine metaphysische Grund-Lage, von der man allenfalls eine intellektuelle Anschauung haben könnte, sondern sie ist - wenn man der Lehre Platons folgt und die transzendentale Bedeutung des kantischen a priori bis zum Äußersten treibt

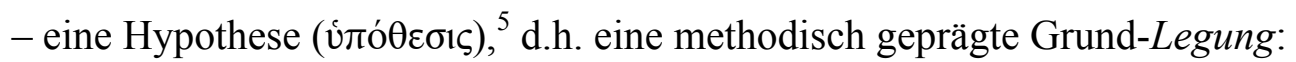

Der sokratische Begriff fragt nur, und weiter geht auch die wohlverstandene Bedeutung des Begriffs nicht. Die Idee dagegen ist das Selbstbewußtsein des Begriffs. Sie ist der Logos des Begriffs; denn sie gibt Rechenschaft vom Begriff. Und im Zusammenhang

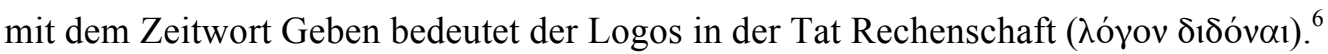

Es handelt sich um das, was Cohen in der Charakteristik der Ethik Maimunis als

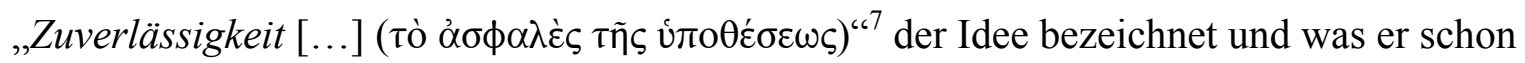

\footnotetext{
${ }^{2}$ Ebd., 15 .

${ }^{3}$ Hermann Cohen, Religion der Vernunft aus den Quellen des Judentums (1919), 2. Aufl. nach dem Manuskript des Verfassers neu durchgearbeitet und mit einem Nachwort versehen von Bruno Strauß (Frankfurt am Main: Kauffmann, 1929), 464.

${ }^{4}$ Vgl. Cohen, Logik der reinen Erkenntnis, 15.

${ }^{5}$ Vgl. ebd., $87 \mathrm{f}$.

${ }^{6}$ Ebd., 15 f.

${ }^{7}$ Vgl. Hermann Cohen, „Charakteristik der Ethik Maimunis“ (1908), in Jüdische Schriften, 3 Bde., mit einer Einleitung von Franz Rosenzweig hrsg. von Bruno Strauß (Berlin: C. A. Schwetschke \& Sohn, 1924),
} Bd. 3, $225 \mathrm{f}$. 
in der zweiten Auflage von Kants Theorie der Erfahrung „die ,Sicherheit der Hypothesis“

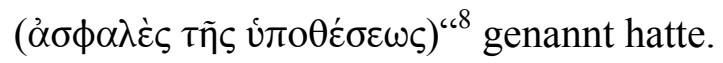

Cohen will den sokratischen Begriff als „Problem“ zum Thema machen und seine logische Bedeutung hervorheben. Denn im sokratischen Begriff tritt nicht nur der Vorrang des Denkens über das Sein, den schon Parmenides erkannt hatte, sondern vor allem die „Grundlegung des Seins im Denken ${ }^{\text {“9 }}$ hervor. Indem Parmenides von einer Identität zwischen Denken und Sein sprach, hatte er Cohen zufolge ,vorzugsweise das Denken durch die Identität charakterisiert [...]. Die Selbigkeit des Seins ist ein Reflex der Identität des Denkens. Diese erst vermag ihre Identität auf das Sein zu erstrecken.“10 Dennoch muss man über Parmenides hinausgehen, auf Pythagoras zurückgehen und die bei ihm mathematisch formulierte Substanz Sokrates folgend zum Begriff erheben, der von sich selbst Rechenschaft geben soll. ${ }^{11}$ Denn die Frage zu stellen: „Was ist?“ verlangt nicht nur die Bestimmung des Inhalts des „Ist“, sondern auch die Begründung der Bestimm-barkeit des Inhalts, d.h. die Begründung des „Was“. Und eben das ist es, was Cohen mit der Frage „Was ist?“ eigentlich meint. Die Frage nach dem Sein, sprich: die „Was ist?“'-Frage, zeigt also keinen Mangel an Inhalt an, sondern sie zielt auf die Bestimmbarkeit dieses Inhalts. So schreibt Cohen:

\footnotetext{
${ }^{8}$ Hermann Cohen, Kants Theorie der Erfahrung (1871, 2. Aufl. 1885, 3. Aufl. 1918), Einleitung von Geert Edel, Bde. 1/1-3 der Werke (Hildesheim/Zürich/New York: Georg Olms, 1987), Bd. 1/1, 22 f.: „Nicht eine etwaige Vermutung ist die Idee, sondern solche Grundlage, welche als Grundlegung eine methodische Basis sichert, und wie in der Geometrie, zu Ergebnissen führt, die von der Voraussetzung geprüft worden, die daher nichtsdestoweniger in der Hypothesis allein gesichert sind“ (ebd., 23, Hervorhebung L.B.).

${ }^{9}$ Cohen, Logik der reinen Erkenntnis, 92.

${ }^{10}$ Ebd., 94.

${ }^{11}$ Vgl. ebd., 29 f.
} 


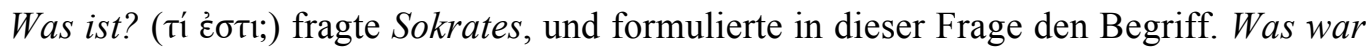

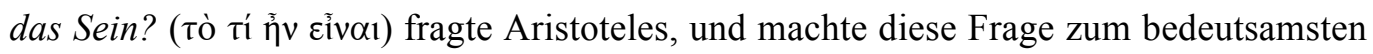
Ausdruck seiner metaphysischen Terminologie. So zeigt sich an diesen hervorragenden Beispielen die logische Bedeutung der Frage, als eines Hebels des Ursprungs [...]. Sie ist der Anfang der Erkenntnis [...]. So ist die Frage die Grundlage des Urteils, man möchte sagen, der Grundstein zur Grundlage. ${ }^{12}$

Das Denken - so kann man zusammenfassen -, das in seiner Selbstbegründung seinen eigenen Inhalt erzeugt, ist für Cohen der wahre und einzige Ursprung der reinen

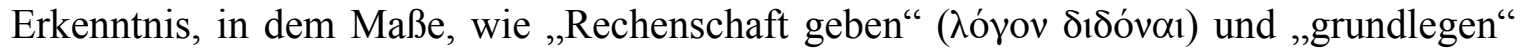

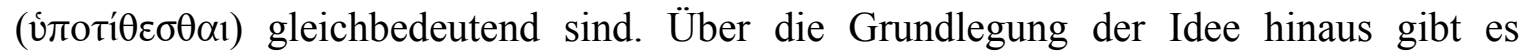
nämlich dem Verfasser der Logik der reinen Erkenntnis nach „keine Wahrheit, keine Erkenntnis und kein Sein; wie es eben auch darunter kein Sein und keine Wissenschaft gibt.“13 Damit begründet Cohen jenes transzendentalmethodische Vorgehen, das hinter jede Art von Voraussetzung zurückfragt und auf diesem Wege die Ausgangsbegriffe von Wissenschaft legitimiert. ${ }^{14}$ Denn die apriorische Grundlegung geht vom Faktum der Wissenschaft aus, um dessen Möglichkeitsbedingungen zu finden: So ist die Erzeugung des Objekts von Seiten des Denkens immer in eine reflektierende Methode eingeordnet und jede ontologische Bedeutung dieser Erzeugung wird ausgeschlossen.

Letztlich zeigt die Frage: „Was ist ,Was ist?’‘“15 auf die unumgängliche

\footnotetext{
${ }^{12}$ Ebd., 83 f.

${ }^{13}$ Ebd., 211.

${ }^{14}$ Vgl. Helmut Holzhey, Cohen und Natorp, 2 Bde. (Basel/Stuttgart: Schwabe \& Co., 1986), Bd. 1, 184 f.

${ }^{15}$ Helmut Holzhey, „Die Vernunft des Problems. Eine begriffsgeschichtliche Annäherung an das Problem
} 
Voraussetzung des Denkens. Diese Voraussetzung ist - so Helmut Holzhey - „eine Voraussetzung des Denkens in doppeltem Sinne: Denken setzt voraus und Denken wird vorausgesetzt. Die Voraussetzung qualifiziert das Denken zum Ursprung der Erkenntnis." "16 Genau hierin sehe ich Cohens entscheidenden Beitrag zur Logik des Idealismus. ${ }^{17}$ Er macht „,das Prinzip des Ursprungs ${ }^{\text {‘18 }}$ zum Fundament seines Systems:

Denken ist Denken des Ursprungs. Dem Ursprung darf nichts gegeben sein. Das Prinzip ist Grundlegung in buchstäblicher Genauigkeit. Der Grund muß Ursprung werden. Wenn anders das Denken im Ursprung das Sein zu entdecken hat, so darf dieses Sein keinen, keinerlei andern Grund haben, als den das Denken ihm zu legen vermag. ${ }^{19}$

Der Unterschied zu Hegel ist aufschlussreich: Dieser hatte den Logos absolut gemacht und ihn mit dem Sein und - am Ende seines Systems - mit der Totalität des Seienden identifiziert; ${ }^{20}$ dagegen gründet Cohen das Sein auf das erzeugende Denken, ${ }^{21}$ oder, mit

der Vernunft“, in Albert Heinekamp, Wolfgang Lenzen und Martin Schneider (Hrsg.), Mathesis rationis. Festschrift für Heinrich Schepers (Münster: Nodus-Publikationen, 1990), 40.

${ }^{16}$ Ebd.

${ }^{17}$ Die zahlreichen Facetten von Cohens Logikkonzept können hier nicht betrachtet werden. Vgl. hierzu Werner Flach, „Hermann Cohens prinzipientheoretisches Logikkonzept“, in Wolfgang Marx und Ernst Wolfgang Orth (Hrsg.), Hermann Cohen und die Erkenntnistheorie (Würzburg: Königshausen \& Neumann, 2001), 99-109.

${ }^{18}$ Cohen, Logik der reinen Erkenntnis, 35.

${ }^{19}$ Ebd., 36.

${ }^{20}$ Vgl. ebd., 329. Zum Unterschied zwischen Cohens „Dialektik des Ursprungs“ und Hegels „Dialektik des Systems“ vgl. Jakob Gordin, Untersuchungen zur Theorie des unendlichen Urteils (Berlin: AkademieVerlag, 1929), 142-68.

${ }^{21}$ Siehe Cohen, Logik der reinen Erkenntnis, 81: „Nur das Denken selbst kann erzeugen, was als Sein 
einem bildlichen Ausdruck gesagt, auf „die schöpferische Souveränität des Denkens““. ${ }^{22}$ Man hat also kein letztlich ontologisches, absolutes Prinzip mehr, sondern nur das reine Denken als ein nicht mehr selbst bedingtes Fundament der Erkenntnis und des Seins: „Die Grundlegung wird Grundlage“, ${ }^{23}$ „,die Tätigkeit selbst ist der Inhalt“, ${ }^{24}$ „,die Erzeugung selbst ist das Erzeugnis. “25 So findet Cohen den „Quell des Etwas“626 im „Nichts“. Dieses ist jedoch kein absolutes Unding, sondern es ist vielmehr als operationeller Begriff zu verstehen, ${ }^{27}$ „um das jedesmalige Etwas, das in Frage steht, in seinem Ursprung, und dadurch erst eigentlich zur Erzeugung und zur Bestimmung zu bringen. ${ }^{628}$

Mit der Frage „Was ist?“ scheint Cohen betonen zu wollen, dass die Philosophie ihre Probleme in einer immer offen bleibenden Form stellt. Bei Rosenzweig spielt diese Frage eine gänzlich andere Rolle. In seinem „Lebenswerk“629 Der Stern der Erlösung gilt die Frage „Was ist?“ sozusagen als Erbsünde, die die ganze philosophische Tradition

gelten darf."

${ }^{22}$ Ebd., 28.

${ }^{23}$ Ebd., 94. Vgl. auch ebd., 116, 305, 428 f., 528.

${ }^{24}$ Ebd., 60.

${ }^{25}$ Ebd., 29.

${ }^{26}$ Ebd., 107.

${ }^{27}$ Vgl. ebd., 105.

${ }^{28}$ Ebd., 89.

${ }^{29}$ So Rosenzweig im Brief an Richard Koch vom 2. September 1928, in Franz Rosenzweig, Briefe und Tagebücher, hrsg. von Rachel Rosenzweig und Edith Rosenzweig-Scheinmann unter Mitwirkung von Bernhard Casper, Bd. 1 von Der Mensch und sein Werk. Gesammelte Schriften (Haag: Martinus Nijhoff, 1979), Teilbd. 2, 1196. 
„von Jonien bis Jena“ ${ }^{\text {(30 }}$ geprägt hat. Diese Frage steht für das Bedürfnis, zu einem letzten Grund zurück zu gelangen. Daher nimmt sie stets die Form einer „Philosophie des All“31 an, d.h. die Form eines allumfassenden, sozusagen totalitären Denkens. Philosophie in diesem Sinn begnügt sich im Unterschied zum unphilosophischen Denken des von Rosenzweig so genannten „gesunden Menschenverstands“ nicht mit dem Sosein eines Dinges. Sie verlangt vielmehr, das „Was ist?“ des Dinges zu entdecken, d.h., für sie muss

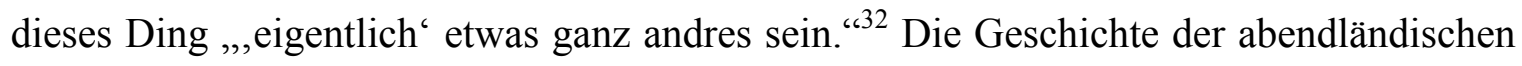
Philosophie erweist sich daher als Folge all der Irrtümer, die aus der Frage „Was ist?“ resultieren. Von ihr getrieben, hatte man im Lauf der Geschichte die Realität - Gott, die Welt und den Menschen insgesamt - zuerst auf die „Welt“ (in der kosmologischen Antike), dann auf „Gott“ (im theologischen Mittelalter) und schließlich auf den „Menschen“ (in der anthropologischen Neuzeit) zurückgeführt. Aber all diese Versuche verbergen nur den entscheidenden Fehler, der bereits in der fragenden Geste des „Was ist?“ selber liegt: die Erwartung, nach dem , ist“ etwas Neues zu finden. ${ }^{33}$ Wie Rosenzweig mit einprägsamer Metapher erklärt,

sind aber diese drei letzten und ersten Gegenstände allen Philosophierens [d.h. Gott, die Welt und der Mensch] Zwiebeln, die man schälen kann, soviel man will, - man kommt

${ }^{30}$ Franz Rosenzweig, Der Stern der Erlösung (1921), Einführung von Reinhold Mayer, Bd. 2 von Gesammelte Schriften (Haag: Martinus Nijhoff, 1976), 13.

${ }^{31}$ Ebd., 5 und passim.

32 Vgl. Franz Rosenzweig, „Das neue Denken. Einige nachträgliche Bemerkungen zum ,Stern der Erlösung،“ (1925), in Zweistromland. Kleinere Schriften zu Glauben und Denken, hrsg. von Reinhold und Annemarie Mayer, Bd. 3 von Gesammelte Schriften (Dordrecht/Boston/Lancaster: Martinus Nijhoff, 1984), 143.

${ }^{33}$ Vgl. ebd. 
immer wieder nur auf Zwiebelblätter und nicht auf etwas „ganz andres“. Nur das Denken gerät notwendig durch die verändernde Kraft des Wörtchens „ist“ auf jene Irrwege. Die Erfahrung entdeckt im Menschen, so tief sie eindringen mag, immer wieder nur Menschliches, in der Welt nur Weltliches, in Gott nur Göttliches. Und nur in Gott Göttliches, nur in der Welt Weltliches, nur im Menschen Menschliches. ${ }^{34}$

Schärfer noch urteilt Rosenzweig in dem posthum erschienenen Büchlein vom gesunden und kranken Menschenverstand - ein Werk, das er selbst für misslungen hielt und daher nicht veröffentlichen wollte. Dort verschiebt er seinen kritischen Akzent vom „ist“ auf das Adverb „eigentlich“, das stets, explizit oder implizit, der „Was ist?“-Frage in der Philosophie beigefügt wird:

„Was ist eigentlich?“, fragt die Frage; das „eigentliche“ Wesen, antwortet die Antwort. „Eigentlich“ - so nämlich fragt und so antwortet kein andrer Mensch als der Philosoph. Im Leben gilt diese Frage so wenig, wie dort die Frage vorkommt. Auch der Philosoph wird sie im Ernstfall nicht stellen. Er wird nicht fragen, was das Viertelpfund Käse „eigentlich“ kostet. Er wird seine Erkorene nicht fragen, ob sie „eigentlich“ seine Frau werden möchte. Er wird nicht bejahen oder verneinen, daß der Angeklagte „eigentlich“ gestohlen habe. Nicht „eigentlich“, sondern „wirklich“ ist das Wort des Lebens. Aber der Philosoph spricht: eigentlich. Indem er seinem Staunen nachgiebt, stehen bleibt, und das Wirkliche sich weiter ohne ihn auswirken läßt, wird er zurückgeworfen und beschränkt auf das Eigentliche. Hier [...] trennen sich seine Wege von den Wegen des gesunden Menschenverstands. Der gesunde Menschenverstand vertraut dem Wirklichen und seinem Wirken. Der Philosoph zieht sich mißtrauisch vor dem fortwirkenden Wirklichen in den geschützten Zauberkreis seines Staunens zurück und versenkt sich in

\footnotetext{
${ }^{34}$ Ebd., 144.
} 
die Tiefe des Eigentlichen. Hier kann ihn nichts mehr aufstören. ${ }^{35}$

Wir haben hier eine Art persönliches j'accuse gegen die Philosophie, die Aristoteles bekanntlich mit dem Staunen über die nächstliegenden Probleme bis zur Entstehung des Alls anfangen lässt. ${ }^{36}$ Die Anklage wird nach Rosenzweig noch dadurch verstärkt, dass jeder Mensch ohne Weiteres zu philosophieren beginnen und somit von einer ,akute[n] Apoplexia philosophica ${ }^{637}$ befallen werden kann. ${ }^{38}$ Die Philosophie ist also nicht nur als solche trügerisch, sondern sie trägt vor allem daran Schuld, dass der gesunde Mensch in eine psychomotorische Lähmung verfällt. Diese Lähmung folgt aus einer besessenen und paralysierenden Fixierung des Verstands - eben à la philosophe - durch die Frage „Was ist?".

Nun ist es eine anerkannte, mit Aristoteles beginnende Tradition, ${ }^{39}$ Sokrates das Verdienst zuzuschreiben, als erster die Frage „Was ist?“ methodisch eingesetzt zu haben, um das Wesen der Dinge zu erkennen. Daher hat man gemeint, Rosenzweigs Kritik richte

\footnotetext{
${ }^{35}$ Franz Rosenzweig, Das Büchlein vom gesunden und kranken Menschenverstand, hrsg. und eingeleitet von Nahum Norbert Glatzer (Düsseldorf: Joseph Melzer, 1964), 31 f. Zu Rosenzweigs Einschätzung des „Büchleins“ vgl. seinen Brief an Hans Ehrenberg vom September 1921 und sein Tagebuch vom 9. Juni 1922, in Rosenzweig, Briefe und Tagebücher, Teilbd. 2, 720 und 793.

${ }^{36} \mathrm{Vgl}$. Aristoteles, Metaphysik, A, 2, 982 b.

${ }^{37}$ Rosenzweig, Büchlein, 57.

${ }^{38}$ Siehe ebd., 33: „Es gibt keinen Gesunden, der vor der Krankheit gefeit wäre. Und im Augenblick, wo sie ihn befällt, im Augenblick, wo der bisher gesunde Menschenverstand meint, philosophieren zu müssen, da gibt es plötzlich keinen größeren ,Eigentlich`-Frager als ihn. Da philosophiert er trotz aller sieben Weisen. Da überphilosophiert er noch den Philosophen. Da gibt es keinen, der sich selber weniger vertraut als er selber. Da ist der gesunde Menschenverstand mit einem Male vom Schlage gerührt.“

${ }^{39}$ Vgl. Aristoteles, Metaphysik, M, 4, 1078 b.
} 
sich implizit vor allem gegen Sokrates. ${ }^{40}$ Es scheint mir aber, dass Rosenzweig mit der Beschreibung der „philosophischen Krankheit“ nur seine grundsätzliche Kritik am „Grunddogma“41 des Idealismus neu formuliert. Dieses Dogma behauptet eine absolute Identität zwischen Sein und Denken, und daher fordert der Idealismus, jedes Objekt zu einem Erzeugnis des Denkens zu machen. Dem setzt Rosenzweig seine drei „letzten Tatsächlichkeiten“42 entgegen, die ihrerseits nicht wechselseitig aufeinander zurückführbar sind. Sie haben einen selbstständigen Status und entziehen sich der idealistischen Totalität. Diese Tatsächlichkeiten sind: erstens der „metaethische“ Mensch, benannt durch Vor- und Zunamen, also das Subjekt der Philosophie; zweitens die „metalogische“ Welt, kontingent und nicht auf die Notwendigkeit der Identität von Sein und Denken zurückführbar; drittens der „metaphysische“ Gott, in seinem Dasein jede Denkbestimmung überschreitend. ${ }^{43}$ Rosenzweigs Kritik an der Identität von Sein und Denken ist auf drei Gedankengänge gestützt. Erstens entlarvt er den tautologischen

\footnotetext{
${ }^{40}$ Vgl. Gianfranco Bonola, „Il disagio della filosofia“, in Franz Rosenzweig, Dell'intelletto comune sano e malato, it. Übers. von Gianfranco Bonola, mit Beiträgen von Gianfranco Bonola und Nahum Norbert Glatzer (Trento: Reverdito, 1987), 170-74. Zum Beleg seiner These zitiert Bonola Franz Rosenzweigs „Anleitung zum jüdischen Denken“ (teilw. veröffentlicht unter dem Titel: „Fragmente aus dem Nachlaß“, 1938/39), in Zweistromland, 601, wo Rosenzweig die „Geschichte des Begriffs (Sokrates - Rückblick auf die vorsokratische Begriffsmetaphysik - Platons ,Idee“ - Kants Kategorie und Idee - Hegels Idee als Kategorie oder der ,konkrete Begriff“)“ zusammenfasst.

${ }^{41}$ Rosenzweig, Der Stern der Erlösung, 165.

${ }^{42}$ Rosenzweig, „Das neue Denken“, 147. Vgl. Franz Rosenzweig, „,Urzelle‘ des Stern der Erlösung. Brief an Rudolf Ehrenberg vom 18.XI.1917“ (1937), in Zweistromland, 136 f. (,reine Tatsächlichkeit“). Vgl. auch Rosenzweig, Der Stern der Erlösung, 25 (,absolute Tatsächlichkeit“), 68 (,schlechthinnige Tatsächlichkeit“), 94 („,bloße“, „feste Tatsächlichkeit“) und passim.

${ }^{43}$ Vgl. Rosenzweig, Der Stern der Erlösung, 10-21.
} 
Charakter $(A=A)$ des formalen Prinzips, also die Selbstbegründung des Denkens (vónбıৎ

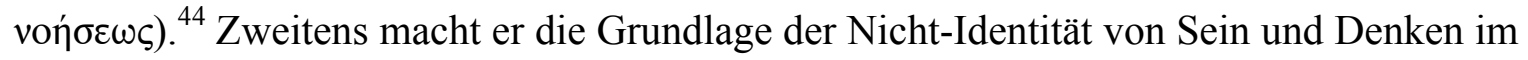
Denken selbst ausfindig, denn dieses Denken kann sich nur deshalb mit dem Sein identifizieren, weil es selbst nichts anderes als die Einheit seiner eigenen Vielheit ist. ${ }^{45}$ Drittens erklärt er, nicht das Sein, sondern das Nichts als Ursprung sei der Ausgangspunkt des Wissens. ${ }^{46}$ Von hier aus schlägt Rosenzweig die Brücke zu Hermann Cohen; dieser habe das Verdienst, mit dem Idealismus gebrochen zu haben:

Und so bauen wir hier, möchte der Meister [Cohen] es auch weit ablehnen, fort auf der wissenschaftlichen Großtat seiner Logik des Ursprungs, dem neuen Begriff des Nichts. Mag er sonst in der Durchführung seiner Gedanken mehr Hegelianer gewesen sein als er

\footnotetext{
${ }^{44}$ Vgl. Rosenzweig, ,„Urzelle‘ des Stern der Erlösung“, 127 f.

${ }^{45}$ Vgl. Rosenzweig, Der Stern der Erlösung, 14.

46 So heißt es im Stern der Erlösung: „Die Philosophie hub erst an, wo sich das Denken dem Sein vermählte. Eben ihr versagen wir, und eben hier, unsre Gefolgschaft. Wir suchen nach Immerwährendem, das nicht erst des Denkens bedarf um zu sein. Deshalb [...] müssen wir das Nichts, wo und wie es uns begegnen mag, aufnehmen und zum immerwährenden Ausgangspunkt des Immerwährenden machen. ,Das ‘ Nichts darf uns nicht Wesensenthüllung des reinen Seins bedeuten, wie dem großen Erben der zwei Jahrtausende Philosophiegeschichte“ (ebd., 22). Rosenzweigs Polemik richtet sich offensichtlich gegen Hegel, beispielsweise gegen dessen Enzyklopädie der philosophischen Wissenschaften im Grundrisse (3. Aufl. 1830), Bde. 8-10 der Werke in zwanzig Bänden, auf der Grundlage der Werke von 1832-1845 neu editierte Ausgabe, Redaktion Eva Moldenhauer und Karl Markus Michel (Frankfurt am Main: Suhrkamp, 1970), Bd. 8, $\S \S 86-88$ : „Das reine Sein macht den Anfang, weil es sowohl reiner Gedanke als das unbestimmte, einfache Unmittelbare ist, der erste Anfang aber nichts Vermitteltes und weiter Bestimmtes sein kann“ (182 f.); „Dieses reine Sein ist nun die reine Abstraktion, damit das Absolut-Negative, welches, gleichfalls unmittelbar genommen, das Nichts ist“ (186); „Das Nichts ist als dieses unmittelbare, sich selbst gleiche, ebenso umgekehrt dasselbe, was das Sein ist"“(188).
} 
zugab - und damit ganz so sehr „Idealist“, wie er es sein wollte -, hier, in diesem Grundgedanken brach er entscheidend mit der idealistischen Überlieferung [...]. Eben zu Hegels Gründung der Logik auf den Begriff des Seins setzte er sich da in entscheidendsten Gegensatz. Und damit wiederum zur ganzen Philosophie, deren Erbe Hegel angetreten hatte. Denn zum ersten Mal erkannte und anerkannte hier ein Philosoph, der - ein Zeichen mehr für die Gewalt dessen, was ihm geschah, - selber sich noch für einen „Idealisten“ hielt: daß dem Denken, wenn es auszog, um „rein zu erzeugen“", nicht das Sein entgegentrat, sondern -Nichts. ${ }^{47}$

Noch aber stellen die drei Tatsächlichkeiten, der „metaethische“ Mensch, die „metalogische“ Welt und der „metaphysische“ Gott, nicht die vom Menschen konkret erfahrene Wirklichkeit dar. Wirklichkeit gibt es nur in den Beziehungen zwischen den drei Elementen: in der göttlichen „Schöpfung“ der Welt, in der „Offenbarung“ Gottes an den Menschen und in der „Erlösung“ der Welt durch den Menschen. So besteht Der Stern der Erlösung, symbolisiert durch den Magen David ( ), aus dem Übereinanderlegen des nach oben gerichteten gleichseitigen Dreiecks $(\triangle)$, das die drei isolierten Elemente darstellt, und des symmetrisch nach unten gerichteten Dreiecks $(\nabla)$, das deren wechselseitige Beziehungen darstellt. ${ }^{48} \mathrm{Um}$ die reale Struktur der Wirklichkeit zu verstehen, muss man die ,alte Philosophie“49 verlassen und sich in Richtung eines „,neuen

\footnotetext{
${ }^{47}$ Rosenzweig, Der Stern der Erlösung, $23 \mathrm{f}$.

${ }^{48}$ Vgl. die Titelseite des Stern der Erlösung, sowie 1, 101, 285, 293. Zur Entstehung des Stern-Zeichens vgl. Rosenzweig, „,Urzelle“ des Stern der Erlösung“, 136 f. und seine Briefe an Margrit Rosenstock vom 22. und 23. August 1918, in Franz Rosenzweig, Die „,Gritli“-Briefe. Briefe an Margrit Rosenstock-Huessy, hrsg. von Inken Rühle und Reinhold Mayer, mit einem Vorwort von Rafael Rosenzweig (Tübingen: BILAM, 2002), 123-25, 127.

${ }^{49}$ Rosenzweig, Der Stern der Erlösung, 116.
} 
Denkens“ bewegen. Rosenzweig nennt es „Sprachdenken“650 oder auch „grammatisches Denken“, ${ }^{51}$ das fähig ist, die Beziehungen ernst zu nehmen und von ihrer Lebendigkeit Rechenschaft zu geben. Denn das alte philosophische Denken ist einsam, will zeitlos sein und kann alles vorwegnehmen, während das neue Sprachdenken des Anderen bedarf, zeitgebunden ist und immer abwarten muss. ${ }^{52}$ Im Passus des Büchlein vom gesunden und kranken Menschenverstand, in dem Rosenzweig seine „neue Philosophie“ zusammenfasst, betont er eben jenes Ernstnehmen der Zeit:

Die neue Philosophie tut $[\ldots]$ nichts andres, als daß sie die „Methode“ des gesunden Menschenverstandes zur Methode des wissenschaftlichen Denkens macht. Worin unterscheidet sich denn der gesunde Menschenverstand vom kranken, der sich, genau wie die alte Philosophie, die Philosophie des „philosophischen Staunens“ - Staunen heißt Stillestehn - in eine Sache verbeißt und sie nicht eher loslassen möchte, bis er sie ganz „hat“? Er kann warten, weiterleben, er hat keine „fixe Idee“, er weiß: kommt Zeit, kommt Rat. Dieses Geheimnis ist die ganze Weisheit der neuen Philosophie. Sie lehrt, mit Goethe zu sprechen, das „Verstehen zur rechten Zeit““.53

So ist es verständlich, dass Rosenzweig, auch wenn er auf den letzten Seiten des Stern der

\footnotetext{
${ }^{50}$ Vgl. Rosenzweig, „Das neue Denken“, 153.

${ }^{51}$ Vgl. ebd., 152.

${ }^{52}$ Vgl. ebd., $151 \mathrm{f}$.

${ }^{53}$ Ebd., 149. Rosenzweig bezieht sich hier auf Johann Wolfgang von Goethe, „Hikmet Nameh. Buch der Sprüche“, in West-östlicher Divan (1819, 2. Aufl. 1827), hrsg. von Konrad Burdach, I. Abt., Bd. 6 der Werke. Weimarer Ausgabe (Weimar: Hermann Böhlau, 1888), 125: „,Warum ist Wahrheit fern und weit? / Birgt sich hinab in tiefste Gründe?` / Niemand versteht zur rechten Zeit! / Wenn man zur rechten Zeit verstünde, / So wäre Wahrheit nah und breit, / Und wäre lieblich und gelinde.“
} 
Erlösung behauptet, dass Gott die Wahrheit sei, ${ }^{54}$ - was implizit ja auch eine Antwort auf die Frage „Was ist Gott?“ darstellt - nicht umhin kann, seine Aussage abzumildern. Die Antwort auf diese per se unmögliche Frage ${ }^{55}$ sage nicht wirklich etwas über das göttliche Wesen aus, sondern zeuge eher von unserer Erfahrung mit der göttlichen Liebe: „Gott ist die Wahrheit - dieser Satz, mit dem wir ein Äußerstes des Wissens zu erschwingen meinten - [...] sagt uns zuletzt doch nichts anderes, als daß er - liebt. “56

Die deutlich hervortretende Differenz zwischen Rosenzweig und Cohen in Bezug auf die Frage „Was ist?“ scheint überraschend, wenn man bedenkt, dass Rosenzweig Cohen seinen ,große[n] Lehrer“57 nannte und ihm das Verdienst zusprach, in der Religion der Vernunft aus den Quellen des Judentums ${ }^{58}$ gänzlich neue Wege des Denkens beschritten zu haben. ${ }^{59}$ Man darf auch nicht vergessen, wie eindrücklich er Cohens Persönlichkeit beschrieben hat, und dies gerade vor dem Hintergrund seiner Rosenzweigs - „Idiosynkrasie“ gegenüber der akademischen Welt, ${ }^{60}$ wo die Professoren

\footnotetext{
${ }^{54}$ Vgl. Rosenzweig, Der Stern der Erlösung, 428 ff.

${ }^{55}$ Vgl. ebd., 434.

${ }^{56}$ Ebd., 432.

${ }^{57}$ Franz Rosenzweig, „Bildung und kein Ende (Pred. 12,12). Wünsche zum jüdischen Bildungsproblem des
} Augenblicks, insbesondere zur Volkshochschulfrage“ (1920), in Zweistromland, 491. Vgl. außerdem Rosenzweig, Der Stern der Erlösung, 281. Vgl. auch, ohne das emphatische „groß“, ebd., 23.

${ }^{58}$ Siehe Anm. 3.

${ }^{59}$ Vgl. Rosenzweig, „Das neue Denken“, 152.

${ }^{60}$ Siehe als Beispiel dafür Rosenzweigs Tagebuch vom 20. November 1906, in Rosenzweig, Briefe und Tagebücher, Teilbd. 1, 65: „Der Professor treibt ein Geschäft, das ihn aus der Welt völlig herausstellt: die reine Wissenschaft. Die Welt dürfte in ihrem eigenen Gebiet eine solche Beschäftigung nur als Sport gelten lassen, nicht als Tagewerk. Deshalb (Der Privatgelehrte - der Eremit der Wissenschaft - wird mitleidig belächelt, der Ordinarius - der Abt im Kloster - bestaunt und bewundert!) gehen die, die einzig einen 
seines Erachtens nicht eigentlich lehren, d.h. im Gespräch mit den Studenten denken, sondern vorwiegend indoktrinieren. Rosenzweig, der auch aus diesen Gründen auf die akademische Karriere verzichtete, die ihm Friedrich Meinecke vorgeschlagen hatte, ${ }^{61}$ schreibt hierzu von einer „Überraschung ohnegleichen“, als er im November 1913 eine Vorlesung Cohens in der Lehranstalt für die Wissenschaft des Judentums in Berlin besuchte:

Gewohnt, auf philosophischen Kathedern kluge Leute zu finden, feinsinnige, scharfsinnige, hochsinnige, tiefsinnige und wie alle die sinnigen Worte heißen mögen, mit denen man den Denker zu loben meint, fand ich einen Philosophen. Statt Seiltänzern, die auf dem gespannten Draht des Gedankens mehr oder weniger kühn, mehr oder weniger geschickt, mehr oder weniger zierlich ihre Sprünge ausführten, sah ich einen Menschen [...]. Hier [...] hatte man das unzerstörbare Gefühl: dieser Mensch muß philosophieren, er hat den Schatz in sich, den das kräftige Wort heraufzwingt. Was ich, irre an der Gegenwart, schon längst nur noch in den Schriften der großen Toten suchte, den streng wissenschaftlichen Geist, der über dem Abgrund einer ungeschiedenen Welt chaotisch drängender Wirklichkeit brütete, hier sah ich es mit

solchen Sport pflegen wollen , abseits', wie früher ins Kloster, so jetzt in die Universitätswelt. Denn diese ist eine Welt für sich. Daraus folgt dann alles übrige: Die begrenzte Anschauung, die sich immer wieder in dem Kreis dieser Wissenschaftswelt (,Gelehrten-Republik`) bewegt, die Überschätzung des eigenen Werts und vor allem des Werts der gelehrten Beschäftigung, der Gelehrtenstolz, ein notwendiger Schutz gegen kritische Blicke, die die Welt von Zeit zu Zeit ins Kloster wirft; nur durch diese gepanzerte Überzeugung vom Wert der Gelehrtenarbeit ist es zu erreichen, daß, wenn draußen vor dem Kloster einer fragt: ,Was tuen sie denn dadrin?` der andere mit tief respektvollem Ernst ihm erklärt: ,Sie beten!‘.“

${ }^{61}$ Vgl. Rosenzweigs Brief an Friedrich Meinecke vom 30. August 1920, in Rosenzweig, Briefe und Tagebücher, Teilbd. 2, 678-82. 
einem Male in sprechender Lebendigkeit, Angesicht zu Angesicht. ${ }^{62}$

Wie soll man also vor dem Hintergrund dieser philosophischen und menschlichen Verehrung für Cohen den Abstand bewerten, der zwischen Rosenzweig und seinem Meister in Bezug auf die Auslegung der Frage „Was ist?“ besteht?

Man hat zum einen die Möglichkeit einer historisch-philologischen Analyse. Sie würde zeigen, dass Rosenzweigs Lektüre der Logik der reinen Erkenntnis eine Art Initialzündung zur Ausarbeitung des Stern der Erlösung war, ${ }^{63}$ und zwar vorwiegend in Bezug auf den Begriff des Nichts, der auch den Ersten Teil in Rosenzweigs Hauptwerk beherrscht. Es würde sich außerdem herausstellen, dass die Hochachtung Rosenzweigs so sehr der Person Cohens galt, dass man geradezu zwischen einem „Cohen imprimens“ dem Cohen, der Rosenzweig beeindruckte - und einem „Cohen impressus“64 - dem Cohen, dessen Werk gedruckt wurde - unterscheiden könnte. ${ }^{65}$ Interessant wäre es auch,

62 Franz Rosenzweig, „Ein Gedenkblatt“ (urspr. unter dem Titel: „Der Dozent. Eine persönliche Erinnerung“, 1917/18), in Zweistromland, 239 (Hervorhebungen L.B.).

${ }^{63}$ Vgl. hierzu bes. Pierfrancesco Fiorato und Hartwig Wiedebach, „Rosenzweig's Readings of Hermann Cohen's Logic of Pure Cognition“, The Journal of Jewish Thought and Philosophy 12, no. 2 (2003): 13946; sowie Dies., „Hermann Cohen im Stern der Erlösung“, in Martin Brasser (Hrsg.), Rosenzweig als Leser. Kontextuelle Kommentare zum „, Stern der Erlösung “ (Tübingen: Max Niemeyer, 2004), 305-55.

${ }^{64}$ So Eugen Rosenstock im Brief an Rosenzweig vom 13. September 1916, in Rosenzweig, Briefe und Tagebücher, Teilbd. 1, 229.

${ }^{65}$ Man müsste sich selbstverständlich fragen, ob Cohen selbst solche Unterscheidung zwischen dem Philosophen, als „Form der Philosophie“ verstanden (so Rosenzweig im Brief an Rudolf Ehrenberg vom 1. Dezember 1917, in ebd., Teilbd. 1, 485), und dem Philosophiegelehrten, d.h. dem Forscher einer spezifischen Wissenschaft, verträte. Es genüge hier, auf die Vorrede zur ersten Auflage seiner Ethik des reinen Willens zu verweisen, wo der Verfasser den Ausspruch behandelt, „,dass der Ethiker ebensowenig ein guter Mensch zu sein brauche, wie der Maler ein schöner“ - so Hermann Cohen, System der Philosophie. 
Rosenzweigs Deutung von Cohens Religion der Vernunft aus den Quellen des Judentums als „Vollendung“ und gleichzeitig „Umkehr“, „Wendung“ oder „Heimkehr“ zu untersuchen - vor allem vor dem Hintergrund von dessen System der Philosophie. ${ }^{66}$ Es ist kein Zufall, dass die Cohen-Forschung dabei immer wieder zu sehr rosenzweigkritischen Ergebnissen kam. Dagegen haben Rosenzweig-Forscher dessen Deutung vielfach als Interpretationsschlüssel verwendet, um das Denken des Begründers der Marburger Schule auszulegen.

Und es bliebe zuletzt noch die Möglichkeit, einen Vergleich zwischen den philosophischen Systemen der beiden Denker im Ganzen anzustellen: desjenigen Cohens, das - wie man mit Rosenzweigs Worten bemerken könnte - ,nach geheiligtem Brauche aus einer Logik, einer Ethik, einer Ästhetik und einer Religionsphilosophie“ ${ }^{\text {667 }}$ besteht, mit demjenigen Rosenzweigs, der sich bewusst ist, dass Der Stern der Erlösung ,außer der

Zweiter Teil. Ethik des reinen Willens (1904, 2. Aufl. 1907), Einleitung auf Englisch von Steve S. Schwarzschild, Bd. 7 der Werke (Hildesheim/New York: Georg Olms, 1981), VIII. Einerseits ficht Cohen das Diktum an, weil der Ethiker seiner Ansicht nach der Idee des Guten immer nachzuspüren habe, andererseits meint er, dass die Sittlichkeit für den Ethiker „,zuvörderst lediglich ein Problem der Erkenntnis werden [muss], in aller der Genauigkeit und Nüchternheit und Sachlichkeit, welche jedes theoretische Problem erfordert“ (ebd.). Denn erst durch dieses wissenschaftliche Untersuchen werde das sittliche Selbstbewusstsein des Ethikers erhöht und allmählich zur Anerkennung der Menschheit, d.h. des höchsten Wertes des menschlichen Daseins, geführt.

${ }^{66}$ Vgl. Franz Rosenzweigs „Einleitung“, in Cohen, Jüdische Schriften, Bd. 1, XIII-LXIV (Zitate XX, XLII); unter dem Titel „Einleitung in die Akademieausgabe der Jüdischen Schriften Hermann Cohens“ auch in Rosenzweig, Zweistromland, 177-223 (Zitate 183, 203).

${ }^{67}$ Rosenzweig, „Das neue Denken“, 141. Neben Cohens Logik der reinen Erkenntnis (Anm. 1), seiner Ethik des reinen Willens (Anm. 65) und seiner Religion der Vernunft (Anm. 3) vgl. Hermann Cohen, System der Philosophie. Dritter Teil. Ästhetik des reinen Gefühls (2 Bde., 1912), Einleitung von Gerd Wolandt, Bde. 89 der Werke (Hildesheim/New York: Georg Olms, 1982). 
vierten dieser Ingredienzien eines ordentlichen Systempunschs [d.h. außer der Religionsphilosophie] die übrigen alle“68 enthält.

Man darf aber in Bezug auf die Frage „Was ist?“ nicht nur die Ferne zwischen Cohen und Rosenzweig konstatieren. Es gibt zwischen ihnen auch eine Nähe, um nicht zu sagen Konvergenz. Wenn man genauer hinschaut, wendet sich Rosenzweigs Kritik an der historischen Rolle der Frage „Was ist?“ vor allem gegen die verändernde Funktion des Wörtchens ,ist“. Er wendet sich also gegen einen überzogenen Anspruch ontologischer Reduktion, den auch Cohen ablehnen würde. Wenn man also die Differenz der beiden genauer fassen möchte, sollte man sich vor Augen führen, was ihre jeweiligen Begriffe von Faktizität und Tatsächlichkeit voneinander unterscheidet, d.h. konkret: Man sollte den Abstand zwischen dem so genannten „Faktum der mathematischen Naturwissenschaft“ bei Cohen und den so genannten „Tatsächlichkeiten“ bei Rosenzweig genau taxieren. In Bezug auf Cohens Faktum bedeutet die Frage „Was ist?“ nämlich die Suche nach den Bedingungen der Möglichkeit des Faktums. Bei Rosenzweigs Tatsächlichkeiten dagegen hat die Frage nicht diese erkenntnistheoretische Relevanz, sondern erfährt ihre eigenen Grenzen durch die gegenseitige Unzurückführbarkeit dieser Tatsächlichkeiten. Um das näher zu erklären, müsste ich jedoch die theoretischen Ansätze der beiden Denker eingehend untersuchen. Das kann ich hier nicht leisten. Dennoch möchte ich zum Schluss auf einige Ähnlichkeiten und Unterschiede aufmerksam machen, die man aus der beiderseitigen Auslegung der Frage „Was ist?“ herauslesen kann.

Wenn es richtig war zu sagen, dass Rosenzweig die Frage „Was ist?“ ausdrücklich im Gegensatz zur philosophischen Tradition versteht, dann scheint es ebenso möglich, fast zwingend, auch die cohensche Interpretation in ein stark

\footnotetext{
${ }^{68}$ Rosenzweig, „Das neue Denken“, 141.
} 
dialektisches Verhältnis zur klassischen Metaphysik zu rücken. Helmut Holzhey folgend hat Pierfrancesco Fiorato zu Recht betont, dass Cohens „verstärkte Frage“, sprich: jene Frage „Was ist ,was ist"?" „an die Stelle der [aristotelischen] metaphysischen Inbesitznahme des Seinsgrundes" tritt. $^{69}$ So behauptet Fiorato:

Wir befinden uns an demjenigen systematischen Ort, an dem sich die Auseinandersetzung Cohens mit der metaphysischen Tradition im Kern vollzieht, und die unergründliche Distanz zum Vorschein kommt, die die „kritische“ Philosophie Cohens von der „,alten Metaphysik“ trennt. Das Urteil des Ursprungs stellt nämlich diejenige Richtung des Fragens dar, die den Grund des Seins betrifft und dadurch einen Zusammenhang zwischen den Urteilsarten hervorbringt. Eine solche Leistung findet jedoch nicht durch die Erfassung des innersten Kerns des Seins statt. Das Urteil des Ursprungs bildet im Gegenteil die Demonstration der Unmöglichkeit einer definitiven Begegnung mit dem Sein im Pol des Wesens. ${ }^{70}$

Wie Rosenzweig, so erkennt auch Cohen an, dass nicht das Sein, sondern das Nichts dem erzeugenden Denken entgegentritt. Aus diesem Grund konnte, wie oben bemerkt, die Logik der reinen Erkenntnis zu einem Initialzünder für die Abfassung des Stern der Erlösung werden.

Genau hier jedoch, wo die Auslegungen des „Was ist?“ zu konvergieren scheinen, divergieren sie zugleich radikal. Cohens Aufmerksamkeit richtet sich auf das „Was“, so dass diese Frage zur Voraussetzung des Denkens, zur Begründung des

\footnotetext{
${ }^{69}$ Pierfrancesco Fiorato, Geschichtliche Ewigkeit. Ursprung und Zeitlichkeit in der Philosophie Hermann Cohens (Würzburg: Königshausen \& Neumann, 1993), 31. Fiorato bezieht sich auf Holzhey, Cohen und Natorp, Bd. 1, 184.

${ }^{70}$ Fiorato, Geschichtliche Ewigkeit, 31.
} 
transzendentalmethodischen Vorgehens werden kann. Dagegen konzentriert sich Rosenzweig auf das ,ist“, welches die Erwartung von etwas ganz anderem auszudrücken scheint, und welches dennoch den Philosophierenden daran hindert, die Wirklichkeit zu erfahren. Auf der einen Seite steht also der kritische Idealismus Hermann Cohens, auf der anderen Seite die „erfahrende Philosophie“, ${ }^{71}$ der „absolute Empirismus“"72 Franz Rosenzweigs. Und wenn Cohen von Rosenzweig her gesehen „mehr Hegelianer gewesen sein [sollte] als er zugab - und damit ganz so sehr ,Idealist", wie er es sein wollte“" ${ }^{73}$ dann könnte man mit einer ähnlichen polemischen Verzerrung, aber ebenfalls nicht ganz zu Unrecht behaupten, dass Rosenzweig mehr „Ontologe“ gewesen sein könnte, als er zugab - und damit ganz so sehr „Empirist“, wie er es sein wollte. Denn mit Cohen, so scheint es Rosenzweig, erhebt sich die Vernunft erneut zum absoluten Prinzip und „der letzte Sinn des Idealismus“ wird noch einmal klar: „Der höchste Gegenstand des Denkens ist das Denken selbst“. ${ }^{74}$ Demgegenüber scheint es mir, dass man bei Rosenzweigs Tatsächlichkeiten eine ontologische Positivität erfährt, die sich den allumfassenden Ansprüchen des totalitären Denkens immer noch entzieht. ${ }^{75}$

\footnotetext{
${ }^{71}$ Rosenzweig, „Das neue Denken“, 144.

${ }^{72}$ Ebd., 161.

${ }^{73}$ Rosenzweig, Der Stern der Erlösung, 23. Vgl. auch seinen Brief an die Mutter vom 15. April 1918, in Rosenzweig, Briefe und Tagebücher, Teilbd. 1, 538, und die Briefe an Margrit Rosenstock vom 18. August 1918 und 21. März 1919, in Rosenzweig, Die „, Gritli“-Briefe, 120 und 257.

${ }^{74}$ Rosenzweig, Der Stern der Erlösung, 160.

${ }^{75}$ Vgl. dazu Luca Bertolino, „Das Nichts und die Philosophie. Rosenzweig zwischen Idealismus und einer Hermeneutik der religiösen Erfahrung“, in Wolfdietrich Schmied-Kowarzik (Hrsg.), Franz Rosenzweigs „,neues Denken“. Internationaler Kongreß Kassel 2004, 2 Bde. (Freiburg/München: Karl Alber, 2006), Bd. 1, pp. 115-20.
} 